\title{
Evaluación Educativa, una Forma de Comenzar por Concretar la Educación Inclusiva
}

\author{
Educational Assessment, a Way to Start Making Inclusive Education a Reality
}

\author{
LUz ARELI Mendoza MÉndez \\ zularelime@hotmail.com \\ https://orcid.org/0000-0001-7909-406X \\ Secretaria de Educación, Veracruz-México
}

\begin{abstract}
RESUMEN:
El presente estudio tuvo como propósito ofrecer información sobre la evaluación educativa y la educación inclusiva como procesos complementarios que implican la aplicación de diferentes técnicas e instrumentos de evaluación basándose en las características de los alumnos. El uso de los resultados y la toma de decisiones con base en dicha información forman parte de la educación inclusiva; sin embargo, si nos referimos a la educación inclusiva ¿por qué no hablar de evaluación inclusiva? Metodológicamente es un estudio teórico de búsqueda sistemática y de análisis de información. Se llegó a concluir que la evaluación educativa y la educación inclusiva son procesos complementarios de aporte de información y apoyos a los docentes y alumnos.
\end{abstract}

\begin{abstract}
:
The purpose of this study was to offer information on educational evaluation and inclusive education as complementary processes that involve the application of different evaluation techniques and instruments based on the characteristics of the students. The use of results and decision-making based on such information are part of inclusive education; However, if we refer to inclusive education, why not talk about inclusive assessment? Methodologically it is a theoretical study of systematic search and information analysis. It was concluded that educational evaluation and inclusive education are complementary processes for providing information and support to teachers and students.
\end{abstract}

\section{Palabras Clave:}

Evaluación; educación inclusiva; diversidad; aprendizaje; evaluación inclusiva.

\section{KEYWORDS:}

Evaluation; inclusive education; diversity; learning; inclusive assessment. 


\section{Introducción}

Todo proceso educativo debe ser valorado existiendo diversas formas para evaluar el desempeño, aprendizajes esperados o propósitos; sin embargo, generalmente se utilizan las mismas técnicas para los alumnos quienes son diferentes, por lo que diversificar las técnicas e instrumentos de evaluación es una forma de atender a la diversidad y empezar a concretar la educación inclusiva dentro del aula. La evaluación es un proceso que, al ser realizado dentro del aula, generalmente guiado por el docente, puede ser modificado conforme a las necesidades, características y nivel de desempeño escolar de los alumnos. Bajo el enfoque de educación inclusiva, estas modificaciones basadas en la diversidad del alumnado son conocidas como ajustes razonables, los cuales son base de la educación inclusiva en el aula.

Además de evaluar el currículo, también se puede valorar la educación inclusiva que se implementa en determinada institución educativa, por lo que la evaluación abarca no sólo aspectos meramente pedagógicos sino institucionales. Para ello existen varios instrumentos, entre los más utilizados y adaptados para México están el Índice de Inclusión de Ainscow y Booth; y GEPIA de Ismael García Cedillo. En este sentido, la evaluación responde a dos procesos fundamentales dentro del actual sistema educativo: atender a la diversidad de alumnado con equidad y fomentar la educación inclusiva, la cual inicia con una autoevaluación y continúa con la toma de decisiones en función de los resultados.

El principal interés en abordar la evaluación en el proceso de enseñanza-aprendizaje, como propuesta para concretar la educación inclusiva en la escuela, se centra en la necesidad que tenemos como docentes de no solo diversificar técnicas e instrumentos de evaluación, sino reflexionar en cómo podemos valorar a la diversidad de alumnos, evitando homogeneizar la evaluación. Esto último debido a que los docentes somo partícipes de la educación inclusiva, debido a la labor que realizamos en diferentes contextos.

Respecto a la evaluación educativa, dentro del proceso de enseñanza y aprendizaje, es fundamental conocer y valorar cómo se está llevando a cabo este proceso. Algunas de las cuestiones que deben ser respondidas, podrían ser, ¿qué han aprendido los alumnos?, ¿fue todo lo que el docente se propuso?, ¿quién decide qué evaluar y cómo evaluar?, entre otras preguntas. No obstante, ¿qué es evaluar?, para responder a la interrogante, primero, se debe de partir delimitando el significado de la evaluación, según lo referido por Mora (2002). Refiere que la evaluación es una acción múltiple y dependerá también del sentido que como escuela y docentes le demos a la evaluación. Así también, la prioridad identificada en materia de evaluación será la quien vaya definiendo el qué, cómo y cuándo evaluar. Ejemplificando, en la educación secundaria, (modalidad secundaria técnica) estas decisiones son tomadas por Academias (docentes organizados de acuerdo con los campos formativos), quienes precisan qué van a evaluar, a través de qué instrumentos y momentos en que lo harán. Luego de una búsqueda exhaustiva, se proponen algunas definiciones de evaluación de acuerdo con diversos autores.

Para Sacristán y Pérez (1996) evaluar hace alusión a un proceso por el cual pueden pasar los alumnos, docentes, contexto escolar y/o áulico, entre otros aspectos, donde reciben atención de quien evalúa, para observar, estudiar y valorar sus características. Esto con base en criterios definidos a fin de emitir un juicio de valor útil al proceso de enseñanza-aprendizaje, donde no solo los alumnos forman parte como evaluados, sino que los diferentes actores educativos e incluso los distintos contextos pueden ser objetos de valoración.

De acuerdo con Santos (2014), la evaluación condiciona el proceso de enseñanza-aprendizaje. He aquí la importancia de cuestionar su origen, finalidad, concreción en el aula y uso de los resultados, es decir, de sus dimensiones éticas, sociales y políticas que lo componen. Además, se debe hacer hincapié en la utilidad y lugar que ocupa la evaluación, pues define cómo será el proceso de enseńanza-aprendizaje. En retrospectiva, se podría afirmar, que desde los parámetros de la evaluación del proceso de enseńanza aprendizaje se busca adecuar, reordenar y eventualmente dirigir el proceso. 
Córdoba (2006) afirma que la evaluación "no puede reducirse solamente a los resultados arrojados por los exámenes que son, en última instancia, una simplificación de la evaluación" (p.2). Se precisa que la evaluación es un proceso gradual, sistemático que va más allá de una prueba escrita u oral presentada por los alumnos pues hace referencia a un conjunto de actuaciones hechas por los docentes para conocer el nivel de competencia curricular de los estudiantes.

Como se puede apreciar, hay coincidencias en las definiciones, los autores definen la evaluación como un proceso, es decir, una serie ordenada de actividades con un fin específico, además se cuestiona la finalidad de la evaluación; así como algunas diferencias. Ejemplificando, Sacristán y Pérez (1996) mencionan que los actores educativos como los contextos pueden ser evaluados, mientras que Córdoba refiere que se evalúa a los alumnos nada más. Cada autor aporta una idea esencial para definir a la evaluación, el primero hace referencia a que al finalizar la evaluación habrá un juicio de valor relevante para la educación; el segundo añade las dimensiones éticas, sociales y políticas; y el tercero menciona que la evaluación no puede reducirse a resultados de exámenes.

Con base en lo anterior, se identifican características de la evaluación como referencia a un proceso que involucra a docentes y alumnos principalmente, aunque no están exentos los demás actores educativos ni los contextos. Dicho proceso valora características y nivel de competencia curricular con la finalidad de emitir un juicio de valor que aporte información relevante para el proceso de enseńanza- aprendizaje.

En cuanto a la evaluación y aprendizaje, si le preguntamos a los docentes qué es evaluar el aprendizaje, probablemente sus respuestas harán referencia a valorar los contenidos revisados durante las clases o lo que marca el Plan de estudios. Por otro lado, si cuestionamos a los estudiantes acerca del mismo tema, encontraremos respuestas con relación a exámenes y/o trabajos por entregar. Desde la experiencia como docente en el nivel de secundaria, los adolescentes resumen la evaluación a pruebas orales o escritas, sin mencionar el proceso que han llevado a lo largo del trimestre o las actividades hechas en clase. Esta afirmación hace que lo vean como algo ajeno a su proceso de enseñanza-aprendizaje y tampoco identifican los momentos evaluativos (evaluación diagnóstica y formativa); sin embargo, la evaluación final o sumativa es la que mejor conocen.

Existen diversas clasificaciones de la evaluación, una de las más conocida y utilizada en el ámbito educativo propone tres momentos: evaluación diagnóstica, formativa y sumativa, las cuales se describen a continuación.

\section{Evaluación diagnóstica}

Al inicio del ciclo escolar es común escuchar a los docentes hablar acerca de cómo realizarán la evaluación diagnóstica para ver como vienen los alumnos. Independientemente del nivel educativo, es una práctica frecuente; los resultados de dicha evaluación sientan las bases para identificar prioridades a trabajar durante el ciclo escolar, le dan la pauta al docente de saber por dónde comenzar. A este proceso, donde la evaluación es específica y diferenciada para cada alumno se le conoce como diagnosis; mientras que, cuando es grupal se le denomina prognosis (Jorba y Casellas, 1997, como se citó en Díaz y Barriga, 2010). De acuerdo con Rosales (1991), la evaluación diagnóstica puede ser de dos tipos: inicial y puntual.

La evaluación diagnóstica, sea individual o grupal, tiene como propósito identificar qué conocimientos tienen los alumnos para poder ajustar los contenidos, metodología y/o estrategias acordes al punto de partida de los alumnos. Esto permitirá identificar las necesidades de aprendizaje de cada estudiante y brindar experiencias de aprendizaje conforme a los resultados de la evaluación diagnóstica.

\section{Evaluación formativa}

La evaluación formativa, de acuerdo con Black y William (2009) es la que "que recolecta evidencia acerca del logro en el aprendizaje y al ser interpretada y utilizada por docentes, estudiantes y sus pares, sirve para tomar decisiones mejor fundadas sobre los próximos pasos a seguir en el proceso de enseñanza aprendizaje" 
(p.8). Esto refiere que forma parte del proceso educativo siendo una de sus principales funciones: la toma de decisiones, no sólo del docente, sino de los alumnos también, haciendo que se involucren en su proceso de aprendizaje.

Por su parte, la Secretaría de Educación Pública de México (SEP, 2013) en su versión digital del documento: Las estrategias y los instrumentos de evaluación desde el enfoque formativo, hace referencia en primer lugar a las habilidades que el alumno debe de desarrollar con el apoyo del docente. Y, en consecuencia, a la función del docente en la evaluación formativa se implementan diversas estrategias e instrumentos de evaluación a lo largo del proceso de enseńanza- aprendizaje y acordes a la diversidad dentro del aula.

\section{Evaluación sumativa}

A la evaluación sumativa también se le conoce como evaluación final, esta evaluación se implementa al final del ciclo escolar. Para Díaz y Barriga (2010) esta contribuye en verificar el grado en que los aprendizajes esperados han sido alcanzados. A través de la evaluación sumativa, los actores del proceso de enseñanza aprendizaje verifican si las acciones de aprendizajes concedidos en los planes y sus propósitos siguieron los criterios y las condiciones previstas. Esta evaluación permite realizar un balance con base en lo propuesto a inicio de ciclo o lo estipulado en el plan de estudios que el alumno debió aprender a lo largo del programa. Siguiendo con estos autores, es importante mencionar la relevancia de las evaluaciones sumativas que han sido asociadas "con la calificación, la acreditación y la certificación; es por ello que muchas veces se le ha confundido con estos temas, especialmente con la acreditación” (Díaz, y Barriga, 2010, p. 352).

En cuanta la evaluación inclusiva, actualmente en educación básica se trabaja con dos Planes de Estudio, el Plan 2011 y el Nuevo Modelo Educativo 2017. En el primero se incorpora la educación inclusiva como un principio pedagógico: favorecer la inclusión para atender a la diversidad, la cual deberá ser pertinente e inclusiva (SEP, 2011); mientras que, en el Nuevo Modelo Educativo 2017(SEP, 2017), la educación inclusiva, además de ser un eje transversal dentro del sistema educativo, se ha diseñado como una estrategia de equidad e inclusión que se debe implementar en el sistema educativo, por lo que la educación inclusiva cobra mayor impacto y relevancia.

Siguiendo con el enfoque de educación inclusiva, se propone que, así como se refiere la necesidad de crear culturas, elaborar políticas y desarrollar prácticas inclusivas, también se implemente una evaluación inclusiva. Esta es definida por Murillo y Duk (2012, p.12) como "un enfoque de la evaluación en los centros educativos comunes en donde la política y la práctica están diseñadas para promover el aprendizaje del alumnado tanto como sea posible", es decir, la evaluación es un proceso sistemático continuo que atiende a todos los alumnos en su proceso de aprendizaje. Siguiendo esa línea, es necesario contar con una propuesta de evaluación "cuya finalidad no puede ser la de clasificar o comparar a los alumnos sino identificar el tipo de ayudas y recursos que precisan para facilitar su proceso educativo" (Duk y Blanco 2012, como se citó en Murillo y Duk, 2012, p. 11). Es conveniente precisar que la evaluación tiene que reivindicar la función principal de dar la pauta para favorecer un proceso de enseñanza-aprendizaje basado en lo que cada alumno necesita, eso de modo, asertivo, equitativo e incluyente.

Para lograr lo anterior, Santiuste y Arranz (2009) proponen que la evaluación debe ser accesible por cuánto una evaluación puntual no parece suficiente para tomar decisiones. Asimismo, los procedimientos de la evaluación deben ser diversos y tener nexo con el currículo escolar. También, se tiene que promocionar la instrumentalidad eficaz de la evaluación en el proceso de seguimiento y logro de metas de aprendizaje. Otros aspectos fundamentales relacionados a la evaluación constituyen, la identificación y el desarrollo de las potencialidades y habilidades. Este exige procesos formativos adecuados previstos en los planes de formación inicial y continua del docente, evitando que el propósito formativo de la evaluación se descuide desde la asunción privilegiada de instrumentos cuantitativos. Además, comunicar a los usuarios los procedimientos de la evaluación como un hecho positivo mediante el cual se pondera el desarrollo del estudiante, evitando la 
burocratización de la evaluación, maximizar la colaboración entre distintos actores, destacar la colaboración de diversos agentes desde la base de la práctica inclusiva.

Finalmente, toda evaluación de implicar al estudiante en todo el proceso debe propiciar la evaluación especializada para sentar una línea de base pertinente, asegurar variados instrumentos de autoevaluación y coevaluación, por mencionar algunos, y garantizar el tiempo de ocupación con la evaluación desde un enfoque cooperativo. Como se puede observar, se hace énfasis en la evaluación formativa como medio para destacar procesos individuales, a fin de guiar la toma de decisiones; además de mantener informados tanto a los alumnos como a los padres de familia del proceso de aprendizaje.

Una situación relevante es el empleo de diferentes recursos y herramientas para evaluar aunado con la identificación de necesidades educativas en el aula, debido a que la aplicación de un instrumento de evaluación aporta información específica de un área, más no de todas. Por ejemplo, para identificar conocimientos de una materia generalmente se emplea una prueba escrita; sin embargo ¿estamos valorando conocimientos o capacidad de retención? Si quisiéramos valorar conocimientos, el Plan y Programas de estudio mencionan la importancia de aplicar esos saberes, por lo tanto, un examen sólo estaría evaluando una parte del aprendizaje, faltaría indagar cómo aplica dichos conocimientos.

En este sentido, se trata de evitar homogeneizar instrumentos de evaluación para permitir valorar el aprendizaje de cada alumno en función de su propio proceso, por lo que se obtendrá información relevante y que sirve de base para el diseño e implementación de las siguientes actividades. Sería muy improbable referirnos a la evaluación si es que no se tiene en cuenta las técnicas e instrumentos para la educación inclusiva, debido a que el proceso de enseñanza-aprendizaje requiere ser evaluado para conocer en qué medida se han alcanzado los aprendizajes esperados, cuáles son las áreas de oportunidad para proponer modificaciones o continuar con lo planeado.

\section{Método}

Metodológicamente el presente estudio es teórico, estado de arte de la cuestión o tema que se utilizó como método la revisión sistemática especializada a fin de realizar la búsqueda, la valoración y la asunción de información relevante sobre la educación inclusiva. Según Aguilera (2014) toda revisión en la investigación científica constituye una forma de investigación que reúne diferentes artículos, tesis y demás investigaciones proporcionando un resumen sobre un tema en especial materia de investigación.

\section{Resultados}

Considerando la conceptualización sobre evaluación, evaluación educativa y educación inclusiva, como procesos complementarios que implican la aplicación de diferentes técnicas e instrumentos de evaluación, basándose en las características de los alumnos y las particularidades del contexto, se ha llegado a sistematizar algunas ideas que se asumen como resultados en nuestra búsqueda. Si bien existe una gran diversidad de técnicas e instrumentos de evaluación, hay algunos que resultan más familiares por su constante uso, por ejemplo, si se quiere saber qué tanto aprendió un alumno de determinada asignatura, se recurre a la aplicación de una prueba escrita, aunque no es el único instrumento de evaluación que permite conocer qué tanto aprendió.

Ante esta situación, la Secretaría de Educación Pública (2013) elaboró una tabla que ilustra los instrumentos que corresponden a cada técnica de evaluación y qué se puede emplear de manera diversificada. Las técnicas e instrumentos de evaluación se pueden clasificar en:

- De observación: guion de observación, registro anecdótico, diario de clase y diario de trabajo. 
- De desempeño de los alumnos: preguntas sobre un procedimiento, cuadernos de los alumnos y organizadores gráficos.

- Para el análisis del desempeño: portafolio de evidencias, rúbrica y lista de cotejo.

- Autoevaluación y coevaluación: reporte del alumno y de sus compañeros.

Conocer las diferentes técnicas e instrumentos de evaluación nos permitirá una mejor intervención docente pues se emplea el instrumento más adecuado para valorar el aprendizaje esperado; sin embargo, no es suficiente conocer las diferentes técnicas e instrumentos para decir que la evaluación llevada a cabo es inclusiva. Dicho proceso requiere de más elementos para responder a la necesidad de aprendizaje de los alumnos, por ejemplo:

- Adaptar la evaluación al ritmo y estilo de aprendizaje del alumno: para ello es imprescindible una evaluación diagnóstica que incluya la identificación de estos elementos.

- Tomar como punto de partida las habilidades y conocimientos del alumno.

- Aplicar diversidad de instrumentos de evaluación.

Si bien no existen instrumentos específicos para realizar una evaluación inclusiva, como se mencionó en el apartado anterior, hay características que se deben tener presente. Por ejemplo, los instrumentos de observación serán un apoyo fundamental en la evaluación inclusiva dado que permite valorar el proceso que el alumno sigue. Se estará identificando sus avances y dificultades en el aprendizaje, que dan la pauta para mejorar la enseńanza. Por su parte, los instrumentos de desempeño, al igual que los de observación, valoran el proceso y, además, pueden ser útiles para la evaluación sumativa porque con ellos se evidencia la aplicación de los conocimientos en diferentes situaciones. Por lo tanto, permite identificar cómo asimiló el alumno lo aprendido y cómo lo aplica; finalmente, la autoevaluación y coevaluación promueven la metacognición que es actor principal en la evaluación, debido a que se asume un rol y responsabilidad mayor dentro de dicho proceso.

Finalmente, en cuanto a la propuesta para evaluar la educación inclusiva en la escuela. Dentro de los textos con mayor relevancia y utilización a nivel internacional tenemos al Índice de inclusión de Booth y Ainscow (2000), el cual es considerado como una guía del proceso de autoevaluación de las escuelas en cuanto a la educación inclusiva. La importancia de la guía estriba en su aporte de tres dimensiones: crear culturas, elaborar políticas y desarrollar prácticas inclusivas. Cada una tiene indicadores y estos a su vez preguntas que van a conducir el trabajo de investigación y evaluación del centro escolar. Para su aplicación en América Latina, se tradujo al español, con las recomendaciones de ajustar los indicadores, preguntas de acuerdo con el contexto y situaciones locales, siempre y cuando se mantenga la esencia de este.

La implementación de prácticas inclusivas dentro del aula busca potenciar las capacidades y habilidades de cada alumno en la medida de sus posibilidades, de trabajar desde la diversidad del alumnado y de necesidades educativas. Por esta razón, se requiere analizar qué elementos están presentes en las prácticas inclusivas para ello, se considera como referencia la Guía de Evaluación de las Prácticas Inclusivas en el Aula (de aquí en adelante: Gepia), dirigida a docentes de escuelas regulares o Centros de Atención Múltiple y su aplicación es individual (Serrato y García, 2014).

La guía consta de dos partes, la primera indaga acerca de las prácticas inclusiva llevadas a cabo por los docentes, y la segunda parte hace referencia a la cultura y políticas de educación inclusiva. No obstante, la que atañe a esta investigación es la primera: prácticas inclusivas que está integrada por cinco componentes básicos presentes en cada aula, en diferentes medidas. Considerando que lo significativo es la frecuencia con que se encuentran y qué resultados se han obtenido a partir de su implementación. Además, nos permite realizar 
un ejercicio de autoevaluación docente para identificar áreas de oportunidades y fortalezas. Adicionalmente, entre los componentes destacan, las condiciones físicas del aula, la planeación, la metodología y la evaluación.

Evaluar y promover una educación inclusiva son procesos complementarios, debido a la valoración de los diferentes contextos donde se desenvuelven los alumnos, para ello utilizan alguna técnica e instrumento de evaluación. Posteriormente, ocurre una toma de decisiones en función de los resultados, luego diseñan estrategias, las implementan y vuelven a valorar, siendo de esta manera un ciclo, donde la evaluación y la educación inclusiva siempre van de la mano.

Por lo anterior, se debe considerar que la evaluación como proceso y resultado "que se realiza en las instituciones está condicionada por diversos agentes que inciden sobre ella” (Santos, 2003, p. 69), por lo que cada proceso de evaluación será único y particular, debido a que sucede en un contexto determinado con características propias. Esa singularidad de la evaluación como proceso y resultado yace conexa con la unicidad del ser humano. Cada estudiante es único, tan particular y singular, por lo mismo, las intervenciones y los instrumentos deben tener esa misma exigencia.

Desde luego, la evaluación vista de ese modo requiere asumir la figura del docente como un agente con situaciones muy particulares, un agente que "actúa en un contexto que condiciona su práctica de la evaluación" (Santos, 2003, p. 69), en ocasiones enfrentado a situaciones difíciles en el que está llamado a conducir y garantizar la evaluación de numerosos estudiantes a su cargo, así como de realizar reportes, en tiempos previstos y según exigencias de los organismos rectores. En todo caso, consideramos que este podría ser uno de los mayores retos enfrentado por los docentes, pues hay grupos de alumnos muy numerosos y en ocasiones no sólo se atiende a un grupo, sino a varios, por ejemplo, los docentes de escuelas secundarias técnicas dan clases a diferentes grupos y grados, por lo tanto, el número de alumnos a evaluar suele ser alto.

Otro reto es utilizar diferentes técnicas e instrumentos de evaluación al reflexionar acerca de nuestra práctica docente. Hemos identificado la aplicación de instrumentos que consideramos pertinentes para valorar los aprendizajes; sin embargo, desde el enfoque de educación inclusiva, los instrumentos deben estar en función de las necesidades y características de los alumnos, no del docente.

Por último, consideramos como otro desafío, el tipo de evaluación que fomentamos dentro del aula y el uso de sus resultados. Precisamos ello, porque generalmente, se le da mayor importancia a la evaluación sumativa por la calificación que se obtiene, para saber si se aprobó o no (función social de la evaluación) y se deja de lado el proceso que se siguió a lo largo del trimestre o ciclo escolar. Por ello, la evaluación inclusiva le da mayor importancia a la evaluación formativa y el proceso que sigue el alumno a lo largo de las actividades, para evidenciar su potencial y progreso.

\section{Conclusiones}

La evaluación educativa es un proceso continuo, al igual que la educación inclusiva, son procesos complementarios puesto que aportan información y apoyo a los docentes y alumnos, aunque esta dependerá del uso dado a los resultados de evaluación y las técnicas e instrumentos empleados. Así también, la evaluación como la educación inclusiva promueven la reflexión del quehacer docente, cuestionan nuestras prácticas dentro del aula y promueven la toma de decisiones basada en el análisis de nuestra práctica.

Si bien existen diversos instrumentos de evaluación, la elección de uno u otro dependerá de qué se quiera valorar en los alumnos, por ejemplo, procedimientos, la comprensión de contenidos, la aplicación de los aprendizajes, sus opiniones y argumentos, entre otros. Si el docente tiene claridad en los objetivos o competencias a valorar será sencillo elegir qué instrumento es el más adecuado para aplicar. Además, será importante retomar el proceso que el alumno ha seguido para alcanzar los aprendizajes, valorar su rendimiento desde el inicio, su trayectoria y resultados en función de sus capacidades y no comparándolas con el resto del grupo, es decir, una evaluación idiográfica. Esto coincide a lo mencionado por Casanova (1997) 
cuando refiere que es una evaluación positiva individualmente porque se centra en el sujeto, en el esfuerzo que pone por aprender y formarse.

También podemos valorar el proceso de educación inclusiva que cada institución educativa promueve. Para ello, existen diversos instrumentos que apoyan la autoevaluación de las escuelas, dentro de los más conocidos están el Índice de inclusión y la guía Gepia, ambas herramientas nos orientan y promueven una retroalimentación para tomar decisiones acordes a las necesidades de los contextos y actores educativos. Finalmente, para eso evaluamos, para conocer dónde estamos, qué podemos hacer y qué se puede cambiar.

\section{Referencias}

Ainscow, M., y Booth, T. (2000). Indice de Inclusión. https://bit.ly/3x19bnk

Black, P., \& Wiliam, D. (2009). Developing the theory of formative assessment [Desarrollando la teoría de la evaluación formativa]. Journal of Personnel Evaluation in Education, 21(1), 5-31. https://bit.ly/2TrUrA9

Blanco, R. (2006). La equidad y la inclusión social: uno de los desafíos de la educación y la escuela hoy. Revista Iberoamericana sobre Calidad, Eficacia y Cambio en Educación 4(3), 1-15 https://bit.ly/3zm32DN

Casanova, M.A. (1997). Manual de evaluación educativa. (2ª ed.). La Muralla. https://bit.ly/3kOsU7v

Córdoba, F. (2006). La evaluación de los estudiantes: una discusión abierta., Revista Iberoamericana, 39(7), 1-9. https:// doi.org/10.35362/rie3972537

Díaz, F., y Barriga, A. (2010). Estrategias Docentes para un Aprendizaje Significativo: una interpretación constructivista. McGraw Hill.

Sacristán, G., y Pérez, A. (1996). Comprender y transformar la enseñanza. Ediciones Morata.

Mora, A. (2004). La evaluación educativa: Concepto, períodos y modelos. Revista Electrónica Actualidades Investigativas en Educación, 4(2), 1-29 https://bit.ly/3rtj8sp

Murillo, F. J., y Duk, C. (2012). Una evaluación inclusiva para una educación inclusiva. Revista latinoamericana de educación inclusiva, 6(1), 11-13 http://hdl.handle.net/10486/662829

Rosales, C. (1991). Estrategias de aprendizaje. Editorial Alianza.

Santos, M. (2003). Dime cómo evalúas y te diré qué tipo de profesional y de persona eres. Revista enfoques educacionales, 5(1), 69-80. https://bit.ly/3BCE1X9

Santos, M. (2014). La evaluación como aprendizaje. Narcea.

Santiuste, V., y Arranz, M. L. (2009). Nuevas perspectivas en el concepto de evaluación. Revista de Educación, (350), 463-476. https://bit.ly/3kELG0V

Serrato, L. T., y García, I. (2014). Evaluación de un programa de intervención para promover prácticas docentes inclusivas. Revista Electrónica Actualidades Investigativas en Educación, 14 (3), 1-25. https://bit.ly/3zlloX4

Secretaria de Educación Pública (2011). Plan de estudios 2011. Educación básica. https://www.gob.mx/cms/uploads/ attachment/file/20177/Plan_de_Estudios_2011_f.pdf

Secretaria de Educación Pública (2012). Las estrategias y los instrumentos de evaluación desde el enfoque formativo. https:// bit.ly/3yapQq9

Secretaria de Educación Pública (2017). Nuevo Modelo Educativo para la Educación obligatoria. https://bit.ly/3kJzGeI 